\title{
EQUALITY CONDITIONS FOR NORM INEQUALITIES IN REPRODUCING KERNEL HILBERT SPACES
}

\author{
AKIRA YAMADA
}

\begin{abstract}
We consider norm inequalities arising from nonlinear maps between reproducing kernel Hilbert spaces. In many cases we know that equality for such inequalities occurs only for the reproducing kernels. To investigate this phenomenon we introduce a new class of RKHSs and give fairly general equality conditions for norm inequalities.
\end{abstract}

Mathematics subject classification (2000): 30D50, 30C85.

Keywords and phrases: reproducing kernel, Hilbert space, norm inequality, tensor product.

\section{REFERENCES}

[1] N. ARONSZAJn, Theory of reproducing kernels, Trans. Amer. Math. Soc. 68 (1950), 337-404.

[2] J. BuRBEA, A Dirichlet norm inequality and some inequalities for reproducing kernel spaces, Proc. Amer. Math. Soc. 83 (1981), no. 2, 279-285.

[3] - Norm inequalities of exponential type for holomorphic functions, Kodai Math. J. 5 (1982), no. 2, $339-354$.

[4] —-, Inequalities for reproducing kernel spaces, Illinois J. Math. 27 (1983), no. 1, 130-137.

[5] - Inequalities for holomorphic functions of several complex variables, Trans. Amer. Math. Soc. 276 (1983), no. 1, 247-266.

[6] L. DE BRANGES AND J. RovNYAK, Square summable power series, Holt, Rinehart and Winston, New York, 1966.

[7] H. M. FARKAS AND I. KRA, Riemann surfaces, Second edition, Springer, New York, 1992.

[8] N. A. LebedeV AND I. M. Milin, An inequality, Vestnik Leningrad. Univ. 20 (1965), no. 19, 157-158. (Russian)

[9] S. SAITOH, Some inequalities for analytic functions with a finite Dirichlet integral on the unit disc, Math. Ann. 246 (1979), no. 1, 69-77.

[10] _ - The Bergman norm and the Szegö norm, Trans. Amer. Math. Soc. 249 (1979), no. 2, 261-279.

[11] _ The Dirichlet norm and the norm of Szegö type, Trans. Amer. Math. Soc. 254 (1979), 355-364.

[12] - Some inequalities for entire functions, Proc. Amer. Math. Soc. 80 (1980), no. 2, 254-258.

[13] — Reproducing kernels of the direct product of two Hilbert spaces, Riazi J. Karachi Math. Assoc. 4 (1982), 1-20.

[14] - A fundamental inequality in the convolution of $L_{2}$ functions on the half line, Proc. Amer. Math. Soc. 91 (1984), no. 2, 285-286.

[15] - Hilbert spaces admitting reproducing kernels on the real line and related fundamental inequalities, Riazi J. Karachi Math. Assoc. 6 (1984), 25-31.

[16] _ - Theory of reproducing kernels and its applications, Longman Sci. Tech., Harlow, 1988.

[17] Natural norm inequalities in nonlinear transforms, in General inequalities, 7 (Oberwolfach, 1995), 39-52, Internat. Ser. Numer. Math., 123, Birkhaüser, Basel, 1997.

[18] S. SCHEINBERG, Uniform approximation by meromorphic functions having prescribed poles, Math. Ann. 243 (1979), no. 1, 83-93.

[19] M. SChIFFER AND D. C. SPENCER, Functionals of finite Riemann surfaces, Princeton Univ. Press, Princeton, N. J., 1954.

[20] M. ZedeK, On the Lebedev-Milin inequality, Proc. Amer. Math. Soc. 33 (1972), 395-397. 